\title{
CONTRIBUCIÓN AL CONOCIMIENTO DE LAS ESPECIES DEL COMPLEJO DE NABIS PUNCTIPENNIS BLANCHARD, 1852 (HEMIPTERA: HETEROPTERA: NABIDAE) EN CHILE
}

\author{
CONTRIBUTION TO THE KNOWLEDGMENT OF THE NABIS PUNCTIPENNIS \\ BLANCHARD, 1852 COMPLEX (HEMIPTERA: HETEROPTERA: NABIDAE) IN CHILE
}

\author{
Eduardo I. Faúndez ${ }^{1,2}$ \& Máriom A. Carvajal ${ }^{1,2}$
}

\begin{abstract}
RESUMEN
Se entregan nuevos datos para las especies del complejo de Nabis punctipennis en Chile, incluyendo nueva información biológica para Nabis faminei, además de nuevos datos distribucionales para esta especie en Magallanes. Se aclara la distribución de Nabis punctipennis en Chile, y se describe una nueva especie del complejo, Nabis ashworthi n.sp., del sur de Chile. Finalmente se entrega una clave para las especies del complejo presentes en Chile.
\end{abstract}

Palabras clave: Heteroptera, Nabidae, Chile, nuevos registros, nueva especie.

\section{ABSTRACT}

New data is provided for the Nabis punctipennis complex in Chile. New biological and distributional data are delivered for Nabis faminei. The distribution of N. punctipennis in Chile is clarified. A new species, Nabis ashworthi is described from Southern Chile. A key to the species of the complex present in Chile is included.

Key words: Heteroptera, Nabidae, Chile, new records, new species.

\footnotetext{
${ }^{1}$ Entomology Department, North Dakota State University, Dept. 7650, P.O. Box 6050; Fargo, ND. E-mail: ed.faundez@gmail. com, mariom.carvajal@gmail.com.

${ }^{2}$ Departamento de Zoología Médica, Centro de Estudios en Biodiversidad (CEBCh), Magallanes, 1979, Osorno, Chile
} 


\section{INTRODUCCIÓN}

Nabidae una familia de Heterópteros predadores, que alberga cerca de 500 especies distribuidas en 20 géneros (Schuh \& Slater, 1995). Los Nábidos se caracterizan por poseer patas protorácicas adaptadas para la captura y el manejo de presas (Schuh \& Slater, 1995). Las especies de esta familia son predadores generalistas de pequeños artrópodos (Lattin, 1989); siendo muchas de ellas consideradas de importancia económica por su acción como controladores de plagas (Braman, 2000). Adicionalmente existen varios reportes de Nábidos picando a humanos, provocando generalmente eritemas y dolor punzante como piquetes de alfiler (Faúndez \& Carvajal, 2011).

Nabidae está representada en Chile por 4 especies Nabis punctipennis Blanchard, 1852 , Nabis (Tropiconabis) consimilis Reuter, 1912, Nabis (Tropiconabis) capsiformis Germar, 1837 y Nabis faminei Stål, 1859 (Volpi \& Coscarón, 2010), siendo N. punctipennis la especie más conocida por su amplia distribución. El complejo de Nabis punctipennis fue definido por Harris (1939), y actualmente contiene 4 especies que se distribuyen en Chile, Argentina, Uruguay y sur de Brasil respectivamente (Cornelis \& Coscarón, 2013). En Chile este complejo está representado por $N$. punctipennis y $N$. faminei. El objeto de la presente contribución es entregar nuevos datos y clarificar situaciones respecto a las especies de este complejo en el país, así como también describir un nuevo taxón perteneciente a este grupo.

\section{MATERIALES Y MÉTODOS}

El material examinado corresponde a las siguientes colecciones: USNM (United States Museum of Natural History, Smithsonian Institution), ACAC (Allan C. Ashworth Collection), EIFC (Eduardo I. Faúndez), IPUM (Instituto de la Patagonia, Universidad de Magallanes). En morfología seguimos a Cornelis \& Coscarón (2013). Las observaciones fueron hechas con ejemplares colectados en el Jardín Botánico "Carl Skottsberg" perteneciente al "Instituto de la Patagonia", de la Universidad

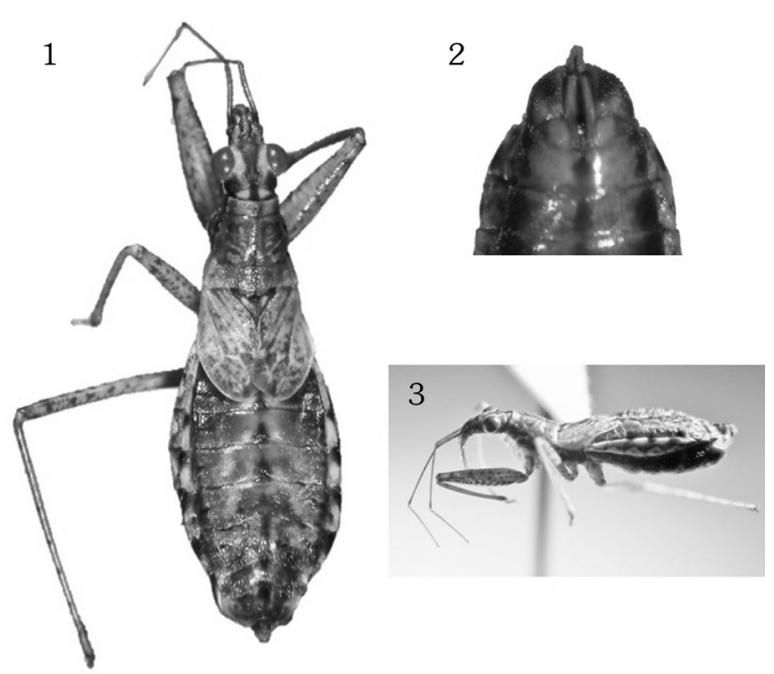

Figs. 1-3, Nabis ashworthi n. sp. 1, habitus; 2, terminalia femenina; 3, vista lateral.

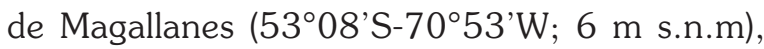
en Febrero 2012. Estos fueron puestos en cajas de madera con cubierta de vidrio, y con trozos de vegetación en la cual las chinches fueron colectadas, mantenidas a una temperatura de $20^{\circ} \mathrm{C}$. Las fotografías fueron tomadas con una cámara digital adaptada a un microscopio estereoscópico. Todas las medidas se encuentran en milímetros.

\section{RESULTADOS}

Nabis ashworthi n. sp. (Figs. 1-3, 10-12)

\section{Descripción}

Características generales: Hembra y macho de similar aspecto. Cuerpo alargado (Figs. 1, 2), cubierto de abundante pilosidad blanca, coloración general amarillo-pardusco con diseños pardos, micróptero (Fig. 1).

Cabeza: Alargada, 1.27 veces más larga que ancha, amarillenta, con una fuerte banda parda por el medio y lados. Ojos muy prominentes rojo oscuro, ocelos rojos ubicados detrás de los ojos. Antenas largas 4-segmentadas, amarillo pardusco, primer antenómero corto, segundo antenómero muy largo (aproximadamente 1,6 veces más largo que el primero y 1.26 veces más largo que el ancho del pronoto, tercer antenómero largo (más 
corto que el segundo y más largo que el primero), cuarto antenómero dañado, la porción remanente es más corta que todos los artejos anteriores. Rostrum robusto, 4-segmentado, primer segmento muy corto, segundo y tercer segmento subiguales (tercero levemente más corto), cuarto segmento corto ( 0.5 veces el tamaño del segundo y tercero),

Tórax: Pronoto levemente más ancho que largo, lóbulo anterior adelgazado, lóbulo posterior con diseños pardos irregulares, presencia de una línea media parda gruesa. Escutelo con forma de triángulo isósceles, con dos fóveas laterales basales y una banda negra en el centro, lados muy claros. Hemiélitros micrópteros (más cortos que el segundo antenómero), no sobrepasando el segundo segmento abdominal, membrana casi imperceptible, coloración clara con escasas y dispersas puntuaciones pardas. Porción ventral de tórax amarillenta con bandas pardas, metapleura rectangular, peritremo ostiolar orientado posteriormente, alargado pasando ligeramente la mitad de la metapleura. Patas amarillentas moteadas irregularmente por manchas pardas circulares. Profémures fuertemente ensanchados con estrías laterales parduscas. Tarsos bisegmentados, segundo tarsómero más largo que el primero. Uñas tarsales largas, color caramelo.

Abdomen: dorsalmente pardusco intercalado con líneas amarillentas, una línea media pardo rojizo que se acentúa alrededor de los vestigios de las glándulas dorsales. Conexiva con borde liso, segmentos conexivales rectangulares, amarillos con un característico diseño pardo rojizo ondeado. Porción ventral del abdomen parda lateralmente y amarilla en el centro, excepto por una irregular línea media parda. Terminalia femenina (Fig.2): Sección genital amplia y ovalada, zona apical muy ensanchada y de borde redondeado en vista ventral, en vista dorsal bordes levemente truncados, primeras gonocoxas compactas, con bordes redondeados, paraterguitos IX amplios y redondeados, primeras gonocoxas delgadas $y$ alargadas, segundas gonocoxas gruesas y alargadas. Genitalia masculina: Pigóforo subcilíndrico (Fig.10), subrectangular en vista ventral (Fig.11), bordes apicales muy truncados, parámeros gruesos, con una amplia escotadura en la sección basal; sección apical levemente producida hacia el interior (e.g. proceso subtriangular y poco aguzado, Fig. 12).
Medidas: Largo total: 6,98; ancho del pronoto: 1,30; alto del pronoto: 1,14 ; largo de la cabeza: 1,20; ancho de la cabeza: 0,95; Diámetro de los ojos: 0,33; espacio interocular: 0,37; ancho del escutelo (incluyendo las fóveas): 0,65; largo del escutelo: 0,47. Antenas: I=0,98, II= 1,64, $\mathrm{III}=1,23$, IV (fragmento) $=0.83$. Rostrum: $\mathrm{I}=0.26$, $\mathrm{II}=0.91, \mathrm{III}=0.99, \mathrm{IV}=0.51$.

Holotipo: Chile, Región de los Ríos, Valdivia, 6km NW Choshuenco, Pte. Blanco 3948'S-72 ${ }^{\circ} 05^{\prime} \mathrm{W}$, Site A, EI, 180m, 5-I-1988, Ashworth, Fuglseth \& Maliscke leg. 19 Valdivian rainforest, on Fuchsia magellanica (USNM).

Paratipos: Chile, Región del Bío Bío, Las Trancas, Chillán, 1-II-2003, Leg. L. Escobar, 10 (en alcohol) (EIFC); Chile, Bío Bío, Lag. El Barco, Guallali, 1100m II-1981, leg. L- E. Peña, 1 o (USNM); Chile. Araucanía, Traiguen, Llico Llana, 8-III-1987, 1 q (USNM).

Etimología: Dedicamos esta especie al Dr. Allan Ashworth (North Dakota State University), en reconocimiento a su trabajo en el sur de Chile, el que le ha permitido elaborar una valiosa colección, la que incluye el holotipo de esta nueva especie.

Discusión:Estaespecieesla primeradecomplejo en presentar microptería, todas las otras tienen formas macrópteras y braquípteras. Nabis ashworthi n. sp. es cercana a $N$. punctipennis y $N$. paranensis y se puede diferenciar de ambas y todo el resto de especies del complejo fácilmente por la proporción de la medida del segundo antenómero y el ancho basal del pronoto. En Nabis ashworthi n. sp., el segundo antenómero es claramente más largo (1.26 veces) que el ancho del pronoto; mientras que en $N$. paranensis estas medidas son subiguales; por otro lado en el resto de las especies del complejo el largo del segundo antenómero es claramente menor que el ancho del pronoto (Cornelis \& Coscarón 2013). Los hemiélitros de $N$. ashworthi n. sp no sobrepasan el segundo segmento abdominal; mientras que en $N$. paranensis (la especie del complejo cuya forma braquíptera posee los hemiélitros más cortos), estas estructuras alcanzan el quinto segmento abdominal. La genitalia de $N$. ashworthi varía de las demás especies del complejo por las siguientes características: la terminalia femenina es más ancha y redondeada apicalmente, teniendo la zona terminal un aspecto semicircular en vista ventral (Fig. 2); carácter que las separa del resto de las especies del complejo, excepto $N$. faminei, de 
la cual se diferencia, por tener esta última los bordes laterales apicales semirrectos; mientras que en $N$. ashworthi $\mathrm{n}$. $\mathrm{sp}$. estos son notoriamente convexos. Por otro lado los parámeros anchos de N. ashworthi n. sp. son muy distintivos dentro del grupo, la única especie que tiene parámeros similares (e.g. gruesos) es $N$. argentinus; no obstante la hoja del parámero de esta última es menos ensanchada, posee un pequeño proceso en la zona anterior basal, la sección apical es notoriamente producida y afilada y se extiende más allá de la zona basal del esclerito; mientras que en $N$. ashworthi n. sp. la sección apical es menos afilada y no se extiende más allá de la base del esclerito; siendo ambos parámeros claramente diferenciables.

Nabis faminei Stål, 1859 (Figs. 4,5, 7, 9)

Este taxón es poco conocido, fue descrito de Magallanes, de Puerto del Hambre, razón a la que probablemente se debe su nombre específico. En varias ocasiones esta especie ha sido cuestionada hasta que Faúndez \& Carvajal (2011) recalcan su validez y mencionan que en Chile estaría restringida a la región de Magallanes. Posteriormente Cornelis \& Coscarón (2013) reafirman su status, dando a conocer por primera vez su genitalia masculina y femenina y entregando detalles de como diferenciarle de $N$. punctipennis, especie con la que suele ser confundida (Faúndez \& Carvajal, 2011). Como mencionamos anteriormente esta especie en Chile se encuentra restringida a la región de Magallanes, mientras que en Argentina se encuentra en Tierra del Fuego y Chubut existe un registro para La Plata en Buenos Aires (Cornelis \& Coscarón, 2013). La biología de esta especie es casi desconocida; China (1963) reporta que se ha colectado manualmente bajo piedras en zonas costeras con presencia de Azorella (Apiaceae), Bolax (Apiaceae), Empetrum (Ericaceae), Pernettya (Ericaceae) y pastos (Poaceae). A continuación entregamos nuevos datos biológicos en base a observaciones e información obtenida de colecciones.

\section{Asociaciones con plantas y actividad predadora}

En el Jardín Botánico Carl Skottsberg en la ciudad de Punta Arenas, Región de Magallanes,
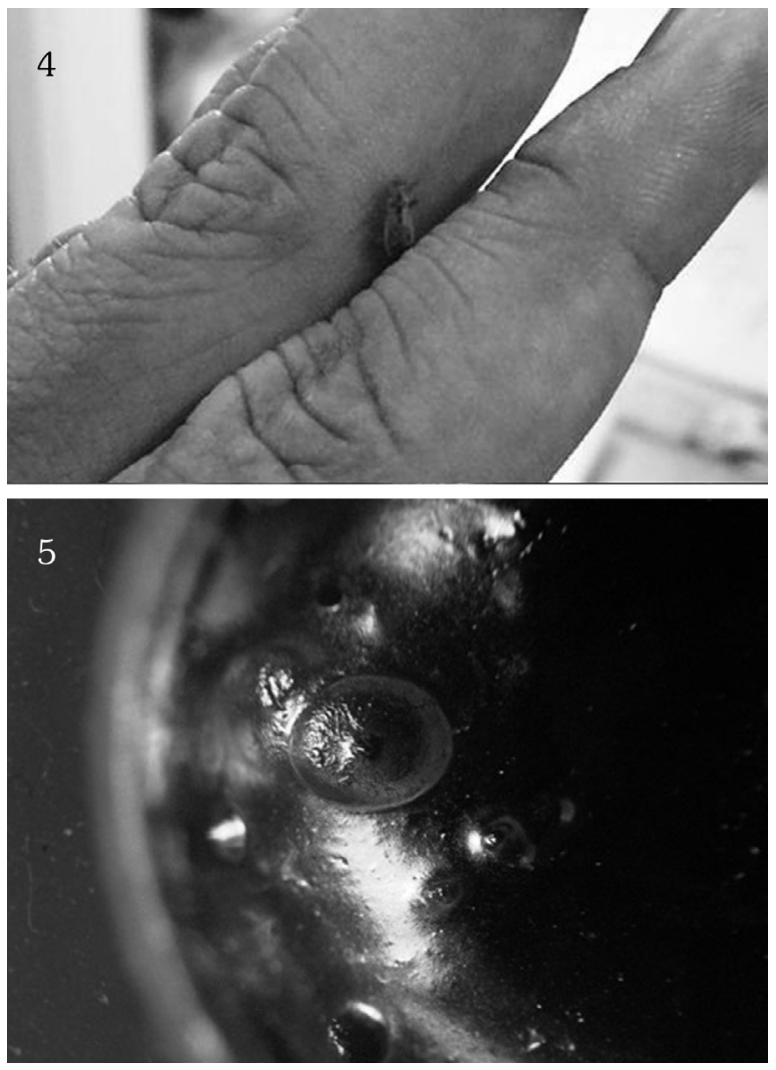

Figs. 4-5. Nabis faminei Stål, 1859. 4, hembra tanteando con aparato bucal en piel humana; 5, marcas de alimentación en recipiente con sangre coagulada.

hemos colectado frecuentemente esta especie en romerillo Chiliotrichum diffusum (G. Forst.) Kuntze (Asteraceae); en este arbusto le hemos observado predar sobre diversas especies no determinadas de Auchenorryncha.

Cerca de la localidad de San Juan, Península de Brunswick, Región de Magallanes, hemos colectado 3 hembras muertas, atrapadas, en la planta insectivora Drosera uniflora Willd (Droseraceae) en una turbera. Junto con las hembras se colectaron también abundantes ejemplares de especies indeterminadas de Auchenorryncha, las que posiblemente intentaban cazar al momento de ser atrapadas.

\section{Picadura y hábitos hematófagos}

En condiciones de laboratorio hemos observado 4 hembras para evaluar su actividad picadora. Al ser molestadas y tomadas con las 
manos no picaban en defensa. Al ser dejadas libremente tendian a recorrer las manos tanteando con su rostrum y buscando los lugares con piel más delgada (Fig.4); 2 hembras lograron picar con éxito en la parte dorsal de las manos, luego de inyectar saliva, succionaron fluidos por unos 10 segundos y luego se retiraron. Los síntomas registrados fueron dolor punzante como piquetes de alfiler por cerca de hora y media y aparición de un pequeño eritema en las zonas de las picaduras.

Para examinar el potencial hematófago de $N$. faminei, 3 hembras, cada una en una caja de crianza fueron provistas de $10 \mathrm{ml}$ de sangre humana como único sustento. Al cabo de 12 horas cada hembra había ingerido gran cantidad de sangre, dejando marcas en el recipiente debido a la acción enzimática sobre la sangre coagulada (Fig.5). Al cabo de 6 horas más las hembras murieron con el abdomen notoriamente distendido, al ser diseccionado se encontró gran cantidad de sangre coagulada en el; el fondo de las cajas de crianza se encontraba repleto de fecas color sanguinoso.

Comentarios: $\mathrm{Al}$ igual que muchas especies de nábidos, $N$. faminei en situaciones de necesidad puede picar de forma adventicia. Schafer (2000) indica que las causas de este tipo de picaduras son principalmente la obtención de agua y solutos. Faúndez \& Carvajal (2011) describen la picadura de Nabis punctipennis, coincidiendo en varios aspectos con los datos aquí entregados; hasta el momento no se han registrado picaduras de especies del complejo punctipennis que envuelvan situaciones de defensa.

\section{Distribución}

En Chile esta especie se conoce solamente de la Región de Magallanes: localidad tipo, Puerto de Hambre, de islas Picton y Navarino (China 1963), Harris (1939) la cita de "Magallanes", y los registros que hemos entregado de San Juan y Punta Arenas; a continuación entregamos los primeros registros para la provincia de Última Esperanza, siendo además la distribución más septentrional conocida para esta especie en Chile:

Material examinado: Magallanes, P.N.T. del Paine, Sector Weber, 13-II-1978, leg. J. Petersen, 3우 1ठ (IPUM); Magallanes Parque Nacional Torres del Paine, Lago Pehoe, 17-II-

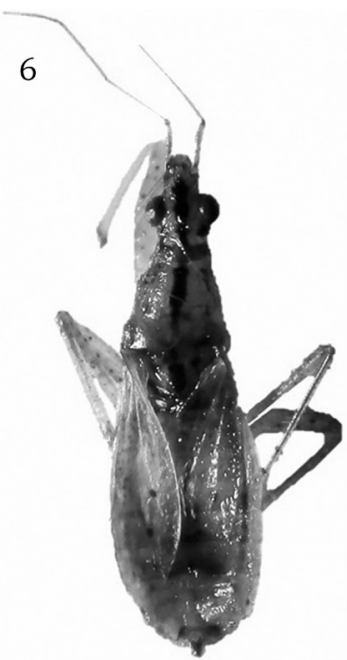

7

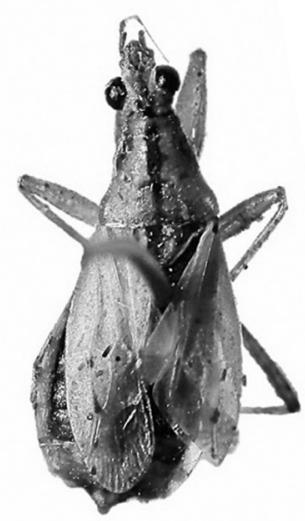

8

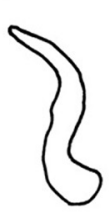

9

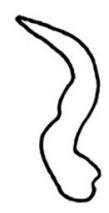

Fig. 6. Nabis punctipennis Blanchard, 1852, habitus (hembra braquíptera); Fig.7. Nabis faminei Stål, 1859, habitus (hembra braquíptera); Fig. 8. N. punctipennis, parámero derecho; Fig.9. N. faminei, parámero derecho.

2012, leg. E. Faúndez 1ð (EIFC); Magallanes, Est. Paine, 20, 21-II-1974, leg. E. Pisano, 1 우 (IPUM); Pr. Última Esperanza, P. N. Torres del Paine, E. side of Lago Pehoé 51 ${ }^{\circ} 06^{\prime}$ S-72 $57^{\circ} \mathrm{W}$, 20-II-1985, leg. A.C. Ashworth, M.J. Gunderson, Steppe-Nothofagus forest, on N. antarctica, 1 ㅇ (ACAC).

Nabis punctipennis Blanchard, 1852 (Figs. 6, 8) =Nabis parvulus Reuter, 1872

Esta especie es la que cuenta con mayor cantidad de referencias en Chile, y ha sido estudiada por diversos autores por su actividad como controlador de plagas (ver Faúndez \& Carvajal, 2011). Sin embargo existen variadas versiones acerca de la distribución de esta especie en Chile, habiendo sido citada desde Antofagasta a Magallanes (e.g. Artigas 1994, Rebolledo et al. 2005, Romero et al. 2007). Faúndez \& Carvajal (2011) descartan la presencia de esta especie en Magallanes, aludiendo a posibles confusiones con Nabis faminei. Hasta ahora no 
se ha revisado en detalle la distribución de esta especie en Chile y sus datos corresponden solo a estimaciones de donde podría encontrarse. De acuerdo los ejemplares estudiados y referencias bibliográficas que incluyen material examinado y no solamente estimaciones, podemos con certeza decir que esta especie se encuentra desde las regiones de Coquimbo hasta la de Los Lagos, incluyendo la Isla de Pascua, sin que su presencia en otras regiones del país haya sido confirmada. Es necesario aclarar también que Gay (1854) en su Atlas, da a esta especie como una especie de Spinola lo que corresponde a un error; ya que en el texto original (Spinola \& Blanchard 1852) lleva una "X", marca que indica a las especies descritas por Blanchard.

Material examinado: Chile: Región de Coquimbo, Los Vilos, II-2008, leg. F. Vargas, 2 우 (EIFC); Chile, Región Metropolitana, El Alfalfal, IV-2009, leg. R. Pérez de Arce, $2 \bigcirc 1$ ô (EIFC); Chile, Región Metropolitana, El Manzano, II-2010, leg. R. Pérez de Arce, $1 \widehat{\jmath}$ (EIFC); Región del Bío Bío, Las Trancas, II-2007,

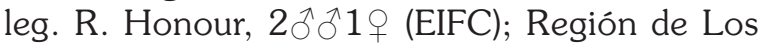
Lagos, Chiloé, III-2002, leg. T. Cekalovic, 1 q (EIFC).

Finalmente para contribuir al reconocimiento de las especies de este complejo en Chile, entregamos la siguiente clave:

Clave para las especies del complejo de Nabis punctipennis presentes en Chile

1(2) Longitud del segundo antenómero notoriamente mayor que el ancho máximo del pronoto, micróptero (Fig.1), parámeros notoriamente ensanchados (Fig.12).

Nabis ashworthi n. sp

2(1) Longitud del segundo antenómero notoriamente menor que el ancho máximo del pronoto, braquíptero (Figs. 6, 7) o macróptero, nunca micróptero, parámeros alargados (Figs 8 y 9).....

3(4) Cuerpo robusto (Fig.7), ojos muy prominentes, abdomen ensanchado apicalmente (Fig.7), hembra con estiloide grueso y robusto, macho con
10

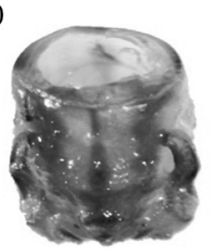

11

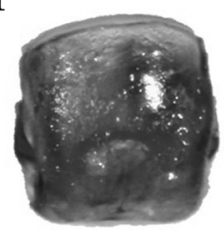

12

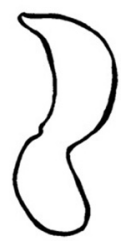

Figs. 10-12. Nabis ashworthi n.sp., 10. pigóforo vista dorsal; 11. pigóforo, vista ventral, 12. parámero derecho.

parámeros robustos y convexos en la zona basal (Fig.9)

Nabis faminei

4(3) Cuerpo menos robusto (Fig.6), ojos menos prominentes, abdomen delgado apicalmente (Fig.6), hembra con estiloide fino, macho con parámeros elongados y comprimidos en la zona basal (Fig.8).

Nabis punctipennis

\section{DISCUSIÓN Y CONCLUSIONES}

El complejo de especies de Nabis punctipennis presenta una amplia distribución en Chile, abarcando desde la Región de Coquimbo hasta la de Magallanes. La distribución en la Región Andina y límites con la Región Neotropical, de las especies de este complejo sugiere un posible origen gondwánico; esta idea se ve reforzada por la semejanza entre las especies del complejo, especialmente la nueva especie aquí descrita, con especies del subgénero de Nabis, Australonabis Strommer, 1988; que se distribuye en Australia y Nueva Zelandia, con las que podría tener un origen común. Es necesario realizar un análisis filogenético en el complejo de Nabis punctipennis para establecer las relaciones entre las especies de este grupo, así como también aclarar su estado taxonómico; ya que tal como las especies australianas, podrían representar un subgénero distinto, o incluso merecer un rango genérico.

\section{AGRADECIMIENTOS}

Agradecemos a Allan C. Ashworth por permitirnos el acceso a su colección, a Thomas Henry por permitirnos el acceso a los ejemplares 
depositados en el USNM, y a María del Carmen Coscarón por atender nuestras consultas.

\section{LITERATURA CITADA}

Artigas, J. M. (1994). Entomología económica: insectos de interés agrícola, forestal, médico y veterinario (nativos, introducidos, y susceptibles a ser introducidos). Vol. 1. Ediciones Universidad de Concepción, Concepción, Chile.

Braman, S. K. (2000). Damsel bugs (Nabidae). Pp. 639-656. In: Schaefer C. W. \& Panizzi A. R. (eds.): Heteroptera of Economic Importance. CRC Press, Boca Raton.

China, W. E. (1963). Hemiptera-Heteroptera collected by the Royal Society expedition to South Chile 1958-1959. Annals and Magazine of Natural History, (13)5[1962]:705-723.

Cornelis, M. \& Coscarón, M. C. (2013). The Nabidae (Insecta, Hemiptera, Heteroptera) of Argentina. Zookeys, 333, 1-30.

Gay, C. (1854). Atlas de la Historia Física y Política de Chile. Tomo II. Imprenta E. Thunot. París.

Faúndez, E. I. \& Carvajal, M. A. (2011). A human case of bitting by Nabis punctipennis (Hemíptera: Heteroptera: Nabidae) in Chile. Acta Entomologica Musei Nationalis Pragae, 51(2), 407-409.

Harris, H. M. (1939). Miscelánea sobre Nabidae
Sudamericanos. Notas del Museo de La Plata. Tomo IV. Zoologia, 26, 368-377.

Lattin, J. D. (1989). Bionomics of the Nabidae. Annual Review of Entomology, 34, 383440.

Rebolledo, O. R., Villegas, G., Klein, C. \& Aguilera, A. (2005). Fluctuación poblacional, capacidad depredadora y longevidad de Nabis punctipennis Blanchard (Hemiptera: Nabidae). Agricultura Técnica, 65, 442446.

Romero, C., Araya, J. E., Guerrero, M. A., Curkovic, T. \& Viñuela, E. (2007). Biología del depredador generalista Nabis punctipennis Blanchard (Hemiptera: Nabidae). Phytoma, 191, 36-44.

Schaefer, C. W. (2000). Adventitious Biters "Nuisance" bugs. Pp. 553-559. In: Schaefer C. W. \& Panizzi A. R. (eds.): Heteroptera of Economic Importance. CRC Press, Boca Raton, Florida.

Schuh, R. T. \& Slater, J. A. (1995). True bugs of the World (Hemiptera: Heteroptera). Classification and natural history. Cornell University Press, New York.

Spinola, M. \& Blanchard, E. (1852). Hemípteros. In: Gay, C. (Ed.) Historia física y política de Chile. Zoología. Vol. 7, 113-320, París.

Volpi, L. N. \& Coscarón M. C. (2010). Catalog of Nabidae (Hemiptera: Heteroptera) for the Neotropical Region. Zootaxa, 2513, 50-68. 
